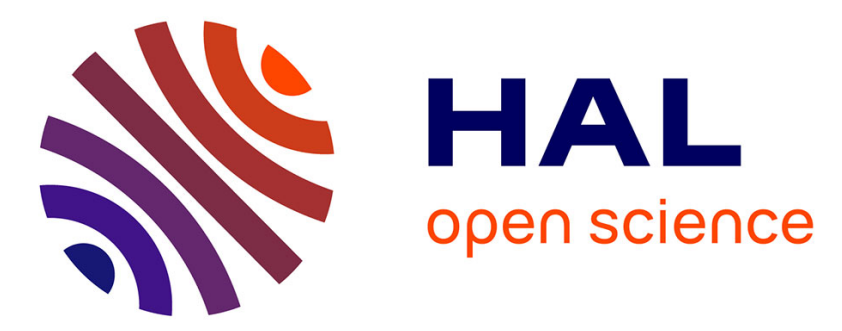

\title{
A 10-moment fluid numerical solver of plasma with sheaths in a Hall Effect Thruster
}

Valentin Joncquieres, François Pechereau, Alejandro Alvarez-Laguna, Anne Bourdon, Olivier Vermorel, Benedicte Cuenot

\section{- To cite this version:}

Valentin Joncquieres, François Pechereau, Alejandro Alvarez-Laguna, Anne Bourdon, Olivier Vermorel, et al.. A 10-moment fluid numerical solver of plasma with sheaths in a Hall Effect Thruster. 54th AIAA/SAE/ASEE Joint Propulsion Conference, 2018, Jul 2018, CINCINNATI, United States. $10.2514 / 6.2018-4905$. hal-02333475

\section{HAL Id: hal-02333475 \\ https://hal.science/hal-02333475}

Submitted on 25 Oct 2019

HAL is a multi-disciplinary open access archive for the deposit and dissemination of scientific research documents, whether they are published or not. The documents may come from teaching and research institutions in France or abroad, or from public or private research centers.
L'archive ouverte pluridisciplinaire HAL, est destinée au dépôt et à la diffusion de documents scientifiques de niveau recherche, publiés ou non, émanant des établissements d'enseignement et de recherche français ou étrangers, des laboratoires publics ou privés. 


\title{
A 10-moment fluid numerical solver of plasma with sheaths in a Hall Effect Thruster
}

\author{
Valentin Joncquieres* \\ SAFRAN AIRCRAFT ENGINES - Campus de l'Espace - 1 avenue Hubert Curien, 27207 Vernon, FR \\ CERFACS - 42, Avenue Gaspard Coriolis, 31057 Toulouse, FR \\ Francois Pechereau \\ LPP, UMR 7648, Ecole Polytechnique, route de Saclay, 91128 Palaiseau Cedex, FR \\ CERFACS - 42, Avenue Gaspard Coriolis, 31057 Toulouse, FR \\ now at ONERA - 29, Avenue de la Division Leclerc, 92320 Chatillon FR \\ Alejandro Alvarez Laguna, Anne Bourdon \\ LPP, UMR 7648, Ecole Polytechnique, route de Saclay, 91128 Palaiseau Cedex, FR \\ Olivier Vermorel, Benedicte Cuenot \\ CERFACS - 42, Avenue Gaspard Coriolis, 31057 Toulouse, FR
}

Electric propulsion can reach higher exhaust velocities compared to chemical systems and thus result in lower propellant mass requirements. Among the different electric propulsion systems, Hall effect thrusters are used for spatial propulsion since the 1970s. However inside a Hall thruster, complex physical phenomena such as erosion or electron anomalous transport which may lower thruster efficiency and lifetime, are not yet fully understood. Thanks to high performance computing, numerical simulations are now considered for understanding the plasma behavior. With the renewed interest for such electric propulsion to supply small satellites, numerical solvers able to predict accurately the real thruster efficiency have become crucial for industry.

This paper presents the approach used and first validation tests of such a solver. The AVIP code solves plasma equations in complex industrial geometries using an unstructured parallelefficient 3D fluid methodology. AVIP also includes a Particle-In-Cell (PIC) solver used as a reference for validation. While full 3D PIC simulations of a Hall thruster still require unaffordable computational time, fluid models provide in a reasonable time 3D results on the plasma behavior inside the discharge channel. In this category, standard drift-diffusion models [1-3] are fast and robust but at the cost of strong hypotheses and simplifications. In particular such models do not describe explicitly the sheath formation in the vicinity of walls and often use analytical models instead. They are limited to simple configurations and only provide a first insight into plasma complex phenomena. The present approach includes a more detailed two-fluid plasma model without drift-diffusion approximation. After the description of the formulation and main features of the solver, the paper focuses on wall boundary conditions which are crucial for the formation of sheaths. It is demonstrated in particular that a vacuum boundary condition is not adapted to fit PIC results. A boundary condition based on wall thermal fluxes is more realistic. The mesh resolution is also found to be critical. The simulation methodology is finally applied to a 2D simulation of a typical Hall effect thruster in order to observe the plasma properties inside the discharge chamber.

*. Ph.D. student. CFD Team at CERFACS; joncquieres@ cerfacs.fr 


\section{Nomenclature}

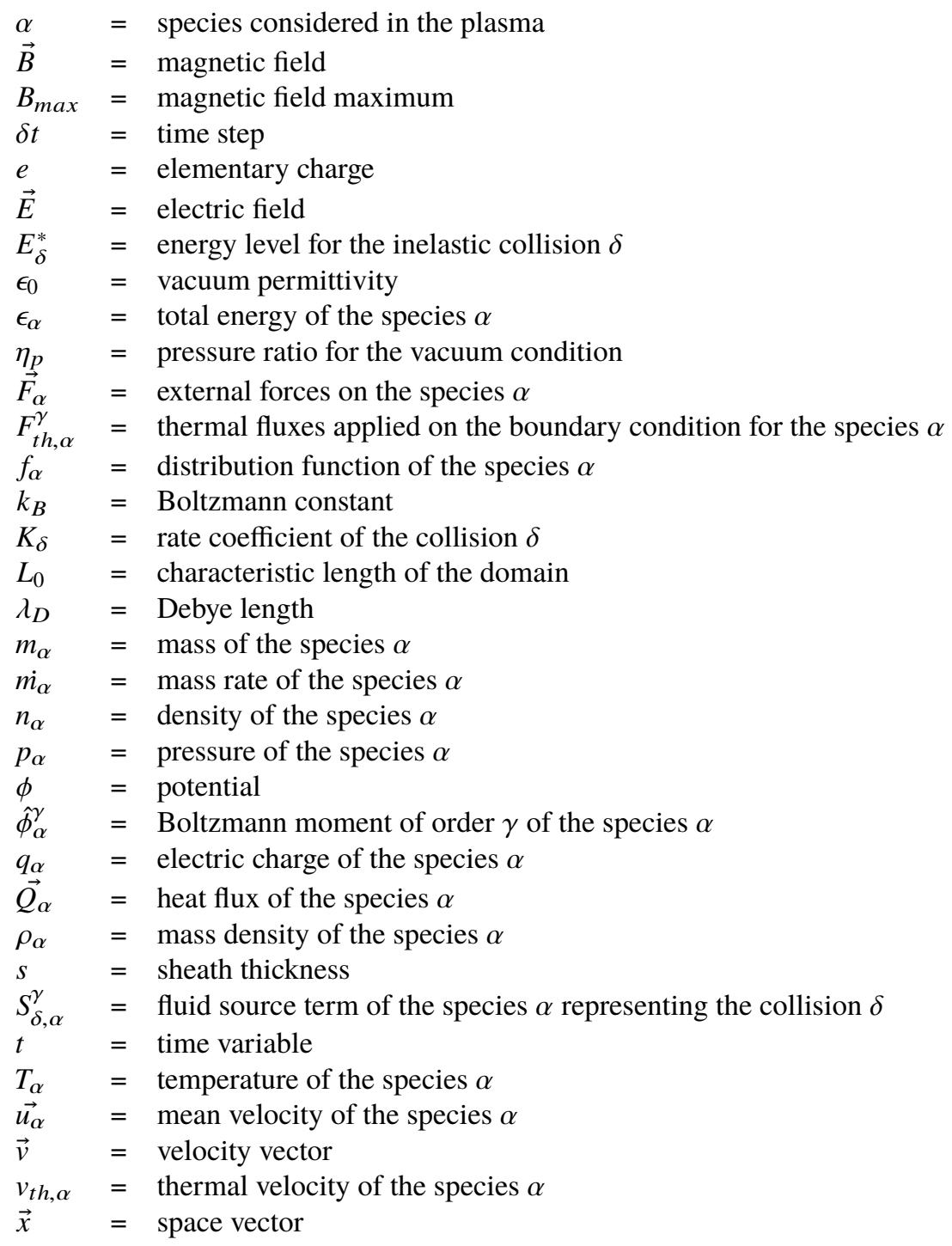

\section{Introduction}

TALL effect thrusters have been massively studied since the 1970 s for satellite propulsion. They are still widely - 1 used to cover a range of applications from satellite station keeping to orbital transfer and positioning. However, the magnetised plasma physics inside these thrusters is particularly complex. Simple analytical models are used for the design of operating parameters suited to the requested mission. Once constructed, the device undergoes several experimental qualifications to demonstrate the thruster efficiency and life time of the device during more than 10,000 hours in a vacuum test facility. These costly and long tests are required because of the lack of insight in Hall thrusters plasma behavior. With the rise of high performance computing, numerical simulation has become attainable and critical for the design of new thrusters in the industrial context.

Accuracy and reliability are of course essential and different numerical plasma models with various orders of accuracy have emerged. On one side, Particle-In-Cell (PIC) models follow the trajectories of a representative number of physical particles called macro-particles in the presence of electromagnetic fields [4, 5]. They are often coupled to a Monte Carlo module to deal with statistical collisions and a Maxwell or Poisson solver to solve electromagnetic fields. On the other side, fluid models describe the statistical behavior of each population of particles. The fluid equations are derived for the statistical moments from the Boltzmann equation supposing a Maxwellian distribution of particles. 
Assumptions can be made such as quasineutrality and equality of ion and electron density, or stationary state neglecting temporal and convective terms, in the so-called drift-diffusion models.

In this paper, a two-fluid detailed model for electrons and ions is presented, without quasineutrality nor drift-diffusion hypotheses. Similarly to [6-10], each species is ruled by a system of Euler equations with source terms representing electromagnetic forces, ionization and other collision processes. The computation of collision source terms follows the idea of Benilov [11, 12]. A Poisson equation is solved for the electric potential. The set of equations has been implemented in the AVIP-Fluid solver, an unstructured parallel-efficient 3D code for the numerical resolution of PDEs. AVIP-Fluid has been validated against several benchmark cases reproducing the main characteristics of a cold magnetised plasma such as plasma oscillation or collision processes. A particular attention is paid on wall boundary conditions as the creation of stable sheaths with correct properties is a difficult challenge for non-quasineutral fluid models. The validated model is then applied to a simplified 2D r-z configuration of Hall thruster. The objective is to analyse the discharge inside a Hall thruster and to demonstrate the capacity of the code for this specific application.

Section III describes the system of fluid equations solved in AVIP. Then, the numerical methods of AVIP are briefly presented in Section IV] with particular focus on the Poisson solver which is a key parameter for good computational performances. In Section $\mathrm{V}$ the sheath formation in a bounded plasma is studied and a numerical strategy to capture them with AVIP-Fluid is detailed. A comparison with AVIP-PIC results is performed to examine the influence of the boundary condition on the sheath formation. Finally, the Section VI shows results about the representative case for Hall effect thrusters.

\section{Physical modeling}

In this section, the fluid model used in AVIP-Fluid is presented. It includes conservative equations for electrons, ions, and neutrals.

Starting from the Boltzmann equation, the evolution of the distribution function $f_{\alpha}(\vec{v}, \vec{x}, t)$ of a plasma species $\alpha$ writes :

$$
\frac{d f_{\alpha}}{d t}+\vec{v} \cdot \vec{\nabla}_{\vec{x}} f_{\alpha}+\frac{\vec{F}_{\alpha}}{m_{\alpha}} \vec{\nabla}_{\vec{v}} f_{\alpha}=\left.\frac{d f_{\alpha}}{d t}\right|_{\text {collisions }}
$$

where $\vec{F}_{\alpha}$ are the external forces exerted on the species $\alpha$ of mass $m_{\alpha}$. A distinction is done between gradient operators $\vec{\nabla}_{\vec{x}}$ in physical space and $\vec{\nabla}_{\vec{v}}$ in phase space, the phases being the velocity components. The left hand side of Eq. 1 represents the collisionless behavior of the species distribution function with convective and time derivative terms and effect of external forces. The right hand side describes the collisional processes.

In a plasma under electromagnetic fields, $\vec{F}_{\alpha}$ is the Lorentz force :

$$
\vec{F}_{\alpha}=q_{\alpha}(\vec{E}+\vec{v} \times \vec{B})
$$

with $q_{\alpha}$ the species electric charge and $\vec{E}$ and $\vec{B}$ the electric and magnetic fields respectively.

In Hall effect thrusters the magnetic field $\vec{B}$ induced by the system is considered negligible compared to the magnetic field imposed by the coils so that $\vec{B}$ is taken constant : $\vec{B}=\vec{B}(x)$. The time evolution of the electric field $\vec{E}$ is no longer governed by the full Maxwell equations but reduces to :

$$
\vec{\nabla} \cdot \vec{E}=\frac{1}{\epsilon_{0}} \sum_{\alpha} q_{\alpha} n_{\alpha}
$$

with $\epsilon_{0}$ the vacuum permittivity and $n_{\alpha}$ the number density of the species $\alpha$. In general the resolution of this equation is done through the electric potential $\phi$ in the domain :

$$
\vec{E}=-\vec{\nabla} \phi
$$

which leads to the Poisson equation :

$$
\Delta \phi=\frac{1}{\epsilon_{0}} \sum_{\alpha} q_{\alpha} n_{\alpha}
$$

Macroscopic properties of each species $\alpha$ correspond to the statistical velocity moments $\hat{\phi}_{\alpha}^{\gamma}$ : 


$$
\begin{aligned}
& \rho_{\alpha}=m_{\alpha} n_{\alpha}=m_{\alpha} \int f_{\alpha} d \vec{v}=m_{\alpha} \hat{\phi}_{\alpha}^{0} \\
& m_{\alpha} n_{\alpha} \vec{u}_{\alpha}=m_{\alpha} \int \vec{v} f_{\alpha} d \vec{v}=m_{\alpha} \hat{\phi}_{\alpha}^{1} \\
& \epsilon_{\alpha}=\frac{3}{2} k_{B} n_{\alpha} T_{\alpha}+\frac{1}{2} m_{\alpha} n_{\alpha}{\overrightarrow{u_{\alpha}}}^{2}=\frac{m_{\alpha}}{2} \int \vec{v}^{2} f_{\alpha} d \vec{v}=m_{\alpha} \hat{\phi}_{\alpha}^{2}
\end{aligned}
$$

Here $\rho_{\alpha}$ is the mass density proportional to the number density $n_{\alpha}$ coming from the $0^{\text {th }}$ order moment $\hat{\phi}_{\alpha}^{0}=n_{\alpha}$. The velocity $\overrightarrow{u_{\alpha}}$ is related to the $1^{s t}$ order moment $\hat{\phi}_{\alpha}^{1}$ and the total energy $\epsilon_{\alpha}$ is the second order moment $\hat{\phi}_{\alpha}^{2}$ which includes both kinetic energy and internal species energy, expressed with the mean temperature $T_{\alpha} . k_{B}$ is the Boltzmann constant.

Integrating the Boltzmann equation in the phase space for each species $\alpha$ and omitting collision terms, the collisionless transport equations are obtained :

$$
\begin{aligned}
\partial_{t} \rho_{\alpha}+\nabla \cdot\left(\rho_{\alpha} \vec{u}_{\alpha}\right) & =0 \\
\partial_{t}\left(\rho_{\alpha} \vec{u}_{\alpha}\right)+\vec{\nabla} \cdot\left(\rho_{\alpha} \vec{u}_{\alpha} \vec{u}_{\alpha}+p_{\alpha} \overline{\bar{I}}\right) & =q_{\alpha} n_{\alpha}\left(\vec{E}+\vec{u}_{\alpha} \times \vec{B}\right) \\
\partial_{t}\left(\epsilon_{\alpha}\right)+\vec{\nabla} \cdot\left(\left(\frac{1}{2} \rho_{\alpha} \vec{u}_{\alpha}^{2}+\frac{\gamma}{\gamma-1} p_{\alpha}\right) \vec{u}_{\alpha}+\vec{Q}_{\alpha}\right) & =q_{\alpha} n_{\alpha} \vec{E} \cdot \vec{u}_{\alpha}
\end{aligned}
$$

These equations are valid for each type of particles, being electrons, ions or neutrals. The fluid pressure $p_{\alpha}$, and the heat flux $\vec{Q}_{\alpha}$ come from higher order moments appearing in the Boltzmann equation and need to be expressed in order to close the system.

The heat flux $\vec{Q}_{\alpha}$ is the third order moment of velocity. An exact closure can be found by integrating even higher order moments of the equations as in [13, 14]. However this only moves the closure problem to higher order moments. Some authors solve a more sophisticated time-dependent equation for the heat flux depending on the magnetic field anisotropy [15]. This approach is not considered in the present fluid model because it introduces three additional equations in 3D to be solved and consequently additional computing time. Instead the system is closed with a simple Fourier law for the heat flux. Previous works [16] have revealed the importance of the electron heat flux in the vicinity of sheaths while the ion heat flux can be considered negligible. The Fourier law for electrons writes :

$$
\vec{Q}_{e}=\frac{\gamma k_{B}^{2} n_{e} T_{e}}{m_{e} n_{n} K_{e n}} \vec{\nabla} T_{e}
$$

with $\gamma$ the heat capacity ratio of the fluid and $K_{e n}$ the electron-neutral elastic collision rate. Ions and electrons are considered as monoatomic particles for which $\gamma=5 / 3$ in three dimensions.

For the pressure $p_{\alpha}$, a perfect gas assumption for each species is made :

$$
p_{\alpha}=n_{\alpha} k_{B} T_{\alpha}
$$

Finally the 10-moment (or 2-fluid) collisionless system for electrons and ions reads :

$$
\begin{aligned}
\partial_{t} \rho_{e}+\nabla \cdot\left(\rho_{e} \vec{u}_{e}\right) & =0 \\
\partial_{t}\left(\rho_{e} \vec{u}_{e}\right)+\nabla \cdot\left(\rho_{e} \vec{u}_{e} \vec{u}_{e}+k_{B} T_{e} n_{e} \overline{\bar{I}}\right) & =-e n_{e}\left(\vec{E}+\vec{u}_{e} \times \vec{B}\right) \\
\partial_{t}\left(\epsilon_{e}\right)+\nabla \cdot\left(\left(\frac{1}{2} \rho_{e} \vec{u}_{e}^{2}+\frac{\gamma}{\gamma-1} k_{B} T_{e} n_{e}\right) \cdot \vec{u}_{e}\right) & =-e n_{e} \vec{E} \cdot \vec{u}_{e} \\
\partial_{t} \rho_{i}+\nabla \cdot\left(\rho_{i} \vec{u}_{i}\right) & =0 \\
\partial_{t}\left(\rho_{i} \vec{u}_{i}\right)+\nabla \cdot\left(\rho_{i} \vec{u}_{i} \vec{u}_{i}+k_{B} T_{i} n_{i} \overline{\bar{I}}\right) & =e n_{i}\left(\vec{E}+\vec{u}_{i} \times \vec{B}\right) \\
\partial_{t}\left(\epsilon_{i}\right)+\nabla \cdot\left(\left(\frac{1}{2} \rho_{i} \vec{u}_{i}^{2}+\frac{\gamma}{\gamma-1} k_{B} T_{i} n_{i}\right) \cdot \vec{u}_{i}\right) & =e n_{i} \vec{E} \cdot \vec{u}_{i}
\end{aligned}
$$


Equations 14 and 17 represent the conservation of ion and electron mass density. Equations 15$] 16$ and 18$] 19$ deal with the conservation of momentum and energy for electrons and ions respectively.

Equation systems (14 16) and (17, 19) without external forces are known as Euler fluid equations. In a plasma, the two sets of Euler equations are coupled through the resolution of the electric field via the Poisson equation and collisions must be added. Collisions between two particles of the same population are considered negligible compared to collisions between different particles. Ionization plays a major role in a Hall effect thruster discharge and needs to be modelled accurately. In the present framework, only elastic collisions of charged particles (ions and electrons) with neutrals are taken into account and Coulomb collisions between charged particles are neglected. Excitation processes are also taken into account to prevent electron energy losses. However we choose not to represent the influence of these processes on neutrals.

With these hypothesis, the system of equations $14 \sqrt{19}$ becomes :

$$
\begin{aligned}
\partial_{t} \rho_{e}+\nabla \cdot\left(\rho_{e} \vec{u}_{e}\right) & =S_{\text {ioniz,e }}^{0} \\
\partial_{t}\left(\rho_{e} \vec{u}_{e}\right)+\nabla \cdot\left(\rho_{e} \vec{u}_{e} \vec{u}_{e}+k_{B} T_{e} n_{e} \overline{\bar{I}}\right) & =-e n_{e}\left(\vec{E}+\vec{u}_{e} \times \vec{B}\right)+S_{e n, e}^{1} \\
\partial_{t}\left(\epsilon_{e}\right)+\nabla \cdot\left(\left(\frac{1}{2} \rho_{e} \vec{u}_{e}^{2}+\frac{\gamma}{\gamma-1} k_{B} T_{e} n_{e}\right) \cdot \vec{u}_{e}\right) & =-e n_{e} \vec{E} \cdot \vec{u}_{e}+S_{e n, e}^{2}+S_{\text {ioniz,e }}^{2}-S_{e x c, e}^{2} \\
\partial_{t} \rho_{i}+\nabla \cdot\left(\rho_{i} \vec{u}_{i}\right) & =S_{i o n i z, i}^{0} \\
\partial_{t}\left(\rho_{i} \vec{u}_{i}\right)+\nabla \cdot\left(\rho_{i} \vec{u}_{i} \vec{u}_{i}+k_{B} T_{i} n_{i} \overline{\bar{I}}\right) & =e n_{i}\left(\vec{E}+\vec{u}_{i} \times \vec{B}\right)+S_{i n, i}^{1} \\
\partial_{t}\left(\epsilon_{i}\right)+\nabla \cdot\left(\left(\frac{1}{2} \rho_{i} \vec{u}_{i}^{2}+\frac{\gamma}{\gamma-1} k_{B} T_{i} n_{i}\right) \cdot \vec{u}_{i}\right) & =e n_{i} \vec{E} \cdot \vec{u}_{i}+S_{i n, i}^{2}+S_{\text {ioniz,i }}^{2}
\end{aligned}
$$

where $S_{\delta, \alpha}^{\gamma}$ is the source term representing, for each species $\alpha$, the collision process $\delta$ (ioniz for ionization, en and in for electron-neutral, ion-neutral elastic collision, exc for excitation). Collision frequencies are generally assumed constant or derived from an analytical solution [17]. In the present paper, collision source terms are computed following the work of Benilov [11, 12], which integrates cross sections of each considered collision depending on the species temperature and mean velocity.

Finally, like in several Hall effect thrusters models [18, 19], neutrals are supposed to be only governed by a mass conservation equation :

$$
\partial_{t} \rho_{n}+\vec{u}_{0, n} \nabla \cdot\left(\rho_{n}\right)=S_{\text {ioniz, } n}^{0}
$$

where $u_{0, n}=[200-300] \mathrm{m}^{-1} \mathrm{~s}^{-1}$ is a constant axial velocity .

The electron-impact ionization is represented by the source term $S_{i o n i z, \alpha}^{0}$ in mass continuity equations 20,23 and 26 The corresponding source terms read :

$$
S_{\text {ioniz,e }}^{0}=S_{\text {ioniz, } i}^{0}=-S_{\text {ioniz, } n}^{0}=K_{\text {ioniz }} n_{n} n_{e}
$$

with $K_{\text {ioniz }}$ the ionization rate coefficient depending on the cross section and the electron energy distribution function. $S_{\text {ioniz, }}^{2}$ and $S_{\text {ioniz, } i}^{2}$ represent the energy transfer between particles associated to the ionization :

$$
S_{\text {ioniz,i }}^{2}=\frac{3}{2} K_{\text {ioniz }} n_{n} n_{e} k_{B} T_{n}, \quad S_{\text {ioniz,e }}^{2}=-n_{n} n_{e} K_{\text {ioniz }} E_{\text {ioniz }}^{*}
$$

with $E_{\text {ioniz }}^{*}$ the energy of the ionization reaction. It assumes that only electrons give their energy for the reaction and neutral energy is transferred to ions. The particle velocity deviation due to ionization is considered negligible in front of elastic collisions.

$S_{\text {exc,e }}^{2}$ in Eq. 22 is the electron energy used to excite neutrals. Several excitation processes are considered :

$$
S_{e x c, e}^{2}=-\sum_{j}^{N} n_{n} K_{e x c, j} E_{e x c, j}^{*}
$$

with $E_{\text {exc,j }}^{*}$ the energy required to excite a neutral at the excited state $j$ and $K_{\text {exc }, j}$ the corresponding reaction rate.

Elastic collisions impact the momentum of particles without creation or exchange of particles, so that $S_{\text {elastic, } \alpha}^{0}=0$. $S_{e n, e}^{1}$ and $S_{i n, i}^{1}$ represent the deviation of particle velocity due to elastic collisions with neutrals : 


$$
S_{e n, e}^{1}=-n_{n} n_{e} K_{e n}\left(u_{e}-u_{n}\right), \quad S_{i n, i}^{1}=-n_{n} n_{i} K_{i n}\left(u_{i}-u_{n}\right)
$$

$S_{e n, e}^{2}$ and $S_{i n, i}^{2}$ express the exchange of energy between particles during an elastic collision :

$$
S_{e n, e}^{2}=-\frac{3}{2} K_{e n} n_{n} n_{e}\left(k_{B}\left(T_{e}-T_{n}\right)+\frac{1}{2}\left(m_{e} u_{e}^{2}-m_{n} u_{n}^{2}\right)\right), \quad S_{i n, i}^{2}=-\frac{3}{2} K_{i n} n_{n} n_{i} k_{B}\left(T_{i}-T_{n}\right)
$$

\section{Implementation and numerical strategy}

Inspired by efficient CFD codes [20, 21], AVIP is a massively parallel code based on a double domain decomposition. First the domain is split in several subdomains using the external library PARMETIS [22]. This decomposition allows a parallel use on a multitude of processors using the Message Passing Interface (MPI). Then each subdomain is divided into cell groups for an easy optimization of the use of processor memory. The AVIP solver handles unstructured 2D triangles and 3D tetrahedral meshes. This ability allows to deal with complex geometries and facilitates mesh adaptation and grid generation.

The numerical resolution of the fluid equations is based on an operator splitting methodology for unstructured meshes. Convection and source terms are integrated separately in time and ensure a first order accuracy resolution. A Strang splitting [23] is also available to get a second-order accurate splitting method. Convective terms are integrated spatially with a finite volume method using a cell-vertex method, with dual cells adapted to unstructured basis. Fluxes at volume interfaces are computed using various Riemann solvers. The HLLC Riemann solver [24] appears to be robust and accurate for the present application and is used in the following. Time integration is performed with a third-order Runge Kutta explicit time scheme in order to avoid large numerical time advancement errors. All source terms (Lorentz forces and collision operators) are solved at once with an implicit time resolution using an external open-source solver from the library ODEPACK [25]. The use of an implicit scheme allows larger time steps especially for the ionization source time integration. Fluid boundary conditions will be detailed in Section $\nabla$

The Poisson equation has elliptic properties and is the most time-consuming step in AVIP. A parallel-efficient solver, the Maphys software [26, 27], developed by the HIEPACS team at INRIA Bordeaux, is used. Maphys is based on a hybrid approach using the same numerical partitioning as the fluid computation. It combines the accuracy of direct methods on each processor and the efficiency of iterative methods to treat the interface system between cores.

\section{Adapted boundary conditions for plasma sheaths}

The length of sheaths is typically a few Debye lengths in a Hall effect discharge chamber. The very thin layer (generally few hundreds of $\mu \mathrm{m}$ ) is crucial for the global behavior of the plasma inside the whole Hall effect thruster. In particular, plasma instabilities triggered at this location, may lead to wall erosion [28, 29] and have a significant impact on the Hall thruster life time. Therefore the evaluation of the electric field and plasma densities inside the sheath is essential to understand and predict the efficiency of a Hall thruster. The physical mechanism of sheaths is fully understood and explained in the literature [30-32]. The loss of electrons at the boundary produces a pressure gradient which accelerates them with larger velocities than ions. The subsequent negative charge difference created at the wall, generates an electric field that accelerates electrons in the direction opposite to their motion, i.e., away from the wall. The balance between electric force and pressure gradient leads to a stationary sheath with a large electric field [33]. The characteristic length of this phenomenon is the Debye length $\lambda_{D}$ defined as :

$$
\lambda_{D}=\sqrt{\frac{\epsilon_{0} k_{B} T_{e}}{n_{e} e^{2}}}
$$

where $T_{e}$ is the electron temperature, $e$ the elementary charge and $n_{e}$ the electron density. The Debye length corresponds to the characteristic length of the electric field induced by a charged particle. The influence on the potential of a charged particle is exponentially dependent on the Debye length :

$$
\phi=\phi_{0} e^{-\frac{x}{\lambda_{D}}}
$$

As a consequence, the Debye length should be resolved to correctly represent the influence of each charged particle on the overall field of electric potential. 
Another standard result of the sheath theory is the Bohm criterion [34] which states that the ion velocity $u_{i}$ inside the sheath must be equal or larger than the ion sound speed to obtain a stable sheath :

$$
u_{i} \geq \sqrt{\frac{k_{B} T_{e}}{m_{i}}}
$$

The equality between the ion velocity and the ion sound speed defines the limit between the sheath and the presheath. This description is valid as long as $\lambda_{D}<<L_{0}$ where $L_{0}$ is the characteristic length of the domain. This condition depends on the electron temperature which must be correctly predicted.

For a sheath with fixed potential, the Child-Langmuir model [35, 36] relates the voltage $\phi_{0}$ across the sheath with the sheath thickness $s$, the electron temperature $T_{e}$ and the Debye length $\lambda_{D}$. For a collisionless sheath, $s$ reads :

$$
s=\left(\frac{4 \sqrt{2}}{9}\right)^{\frac{1}{2}}\left(\frac{e \phi_{0}}{k_{B} T_{e}}\right)^{\frac{3}{4}} \lambda_{D}
$$

This relation is based on fluid macroscopic properties of ions and electrons, i.e., assuming a Maxwellian distribution function, and neglects the electron space charge.

Contrary to fluid solvers, the boundary condition for creating sheaths near walls in a PIC solver is quite simple. Each particle (ion or electron) exiting the domain is lost at the wall boundary which naturally leads to a stationary sheath at the wall or electrode.

Creating stable sheaths in fluid models is less straightforward. By construction, quasineutral solvers do not solve the sheaths and replace the sheath by equivalent boundary conditions [19]. Analytical models [37-41] for the sheath and the presheath, that impose potential drop and plasma densities are commonly used.

On the other hand, drift-diffusion solvers coupled to a Poisson equation are able in principle to represent sheaths [42, 43]. The wall flux can be expressed considering that a charged particle hitting a wall is instantaneously neutralized and assuming that no particles are coming from the wall. Particles may exit at the wall due to their thermal motion and not only their mean velocity. This corresponds to the particles of the Maxwellian distribution function that have a positive (or negative) velocity fluctuation.

The present model is a 10-moment fluid formulation with Poisson equation. Imposing boundary conditions on such equation system is a crucial issue to reproduce correctly the sheath formation. Following the work of Cagas and Hakim [16], it is possible to create sheaths near walls assuming a vacuum just outside the domain for ions and electrons. This means that each charged particle at the wall is instantaneously neutralized and lost.

In [17, 44], wall boundary conditions are expressed using explicit fluxes crossing the boundary, including the flux due to particle thermal motion, as drift-diffusion solvers. In this way, the half of the Maxwellian distribution representing particles that exit the domain, is considered as an outgoing flux.

In the following, a PIC simulation is first performed with the AVIP-PIC solver in order to understand the formation of the sheath and the behavior of macroscopic plasma properties. Note that the AVIP-PIC solver uses a Boris scheme for the transport of particles without collisions and in the presence of an electric field. Secondly, PIC results are considered as the reference and two different boundary conditions with AVIP-Fluid are compared with. Finally the influence of the mesh resolution on AVIP-Fluid results is addressed.

\section{A. Test case on a collisionless sheath : preliminary results with AVIP-PIC}

In this section the formation of a 2D collisionless anode sheath in a Xenon gas is studied $\left(m_{X e}=2.1810^{-25} \mathrm{~kg}\right)$ with the AVIP-PIC solver. The AVIP-PIC code is used as a reference to highlight the balance between pressure gradient and Lorentz force. The y direction is considered periodic. The anode potential $\phi_{0}$ at $x=0$ is set to $200 \mathrm{~V}$ and the cathode potential to $\phi_{L}=0 V$ at $x=L$. No secondary electron emission or other wall interaction are considered. Electron particles are initialized with a Maxwellian velocity distribution function at an initial temperature of $T_{e, 0}=10 \mathrm{eV}$. Cold ions are initially at $T_{i, 0}=0.1 \mathrm{eV}$. Initially, 50 macro-particles per cell are set to ensure statistical convergence. Macro-particles weights are initialized in order to get same electron and ion densities of $n_{e}=n_{i}=5.010^{16} \mathrm{~m}^{-3}$. A fixed timestep of $\Delta t=510^{-12} \mathrm{~s}$ is used for the simulation. The Debye length computed with these initial conditions is $\lambda_{D}=105.2 \mu \mathrm{m}$ and is the reference length for all simulation cases.

After $100 \mathrm{~ns}$ the sheath starts to form as evidenced by ions and electrons densities in Fig. 1a The electron density decreases faster than the ion density due to the high mobility of the much lighter electrons. The thickness of the sheath 
represents the area where the plasma is not quasineutral and is around 8 Debye lengths at $t=0.1 \mu \mathrm{s}$. Also shown in Fig. 1a the electron temperature slightly increases due to the presence of a strong electric field in Fig. $1 \mathrm{~b}$.

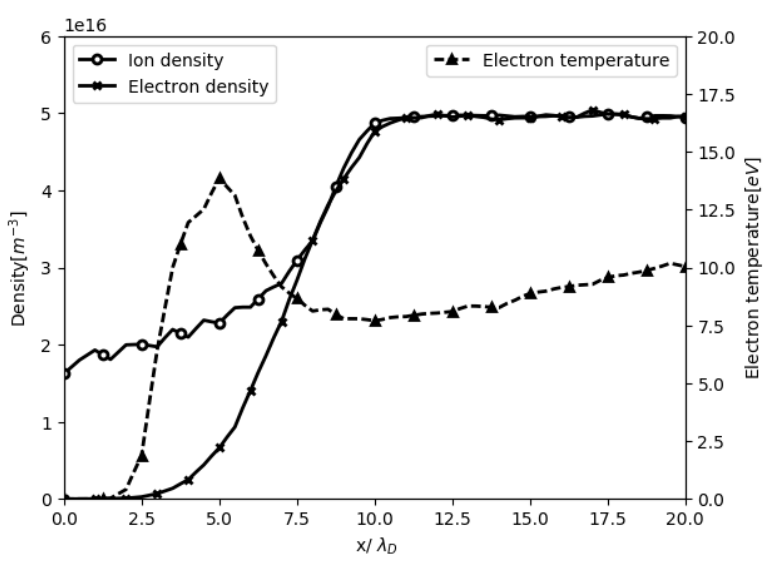

(a) Densities and electron temperature

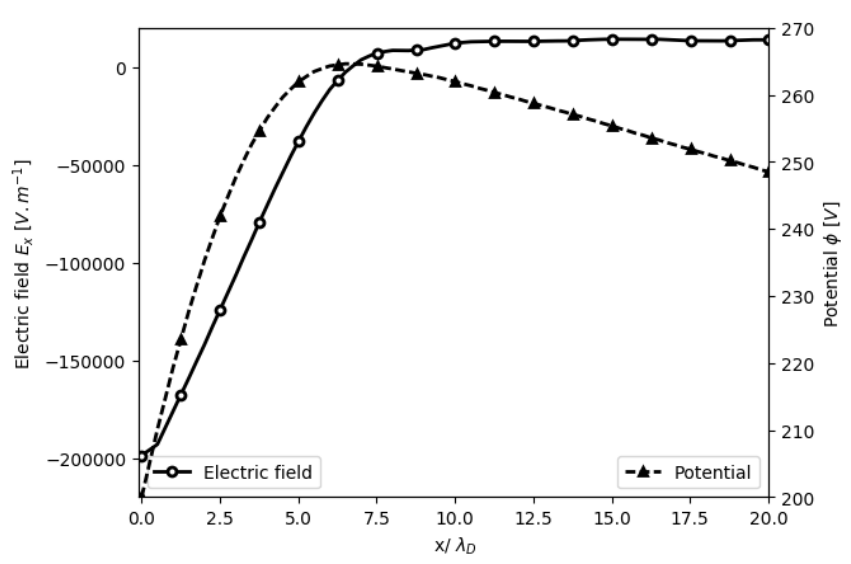

(b) Potential and electric field

Figure 1 Characterization of the plasma inside the anode sheath at $t=0.1 \mu \mathrm{s}$, AVIP-PIC simulation

The electric field magnitude increases in the sheath due to the source term proportional to $\left(n_{i}-n_{e}\right)$ in the Poisson equation, Eq. 55 The potential, fixed at the anode, is shifted in the sheath by $65 \mathrm{~V}$ at $t=0.1 \mu \mathrm{s}$. This drop of potential is higher than the expected value at steady state, as the low mobility of ions induces long transient states.

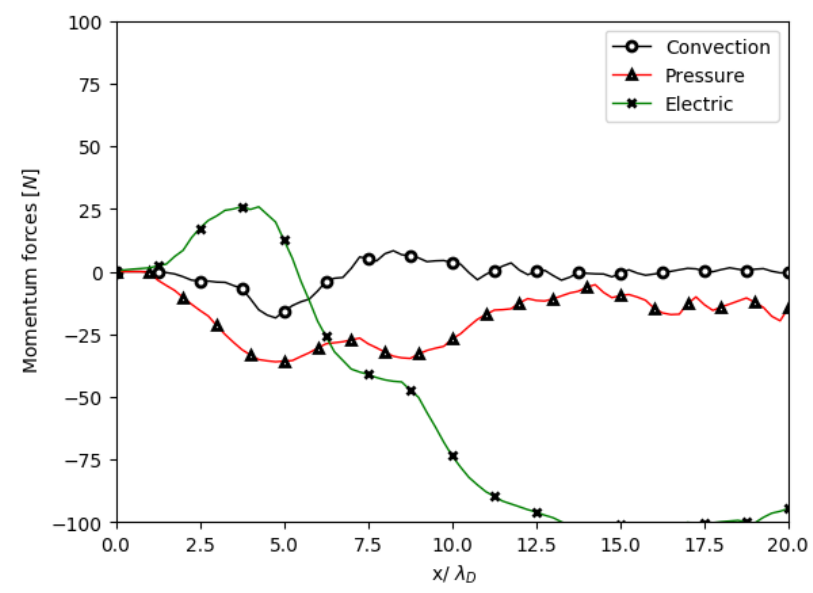

FigUre 2 Balance between momentum equation forces inside the sheath at $t=0.1 \mu \mathrm{s}$, AVIP-PIC simulation

Figure 2 represents the different contributions to the electron momentum conservation equation derived from the PIC simulation. The convection term is negligible inside the whole domain and particularly in the sheath. As mentioned in the literature, the sheath results from the balance between pressure gradient and electric forces. Electrons exit the domain and create a negative pressure gradient. This pressure gradient tends to impose a negative electron velocity meaning that the force expulses the electrons out of the domain. The negative electric field created by the charge difference induces a positive Lorentz force and a negative electron velocity. At $t=0.1 \mu \mathrm{s}$, the electric force is not strong enough to neutralize the pressure gradient and to stabilize the sheath. In the presheath, the Lorentz force is even negative. The steady state is not reached.

At equilibrium the plasma sheath stabilizes with characteristic potential drop and thickness. In the present case this is obtained at $t=0.3 \mu \mathrm{s}$ as shown in Fig. 3. The sum of momentum forces near the wall is slightly negative meaning that electrons still leave the domain. The net force at the wall is around $5 \mathrm{~N}$. In the presheath, the electric force is now positive and compensates the pressure gradient. For an anode sheath, this part of electrons exiting the domain through 


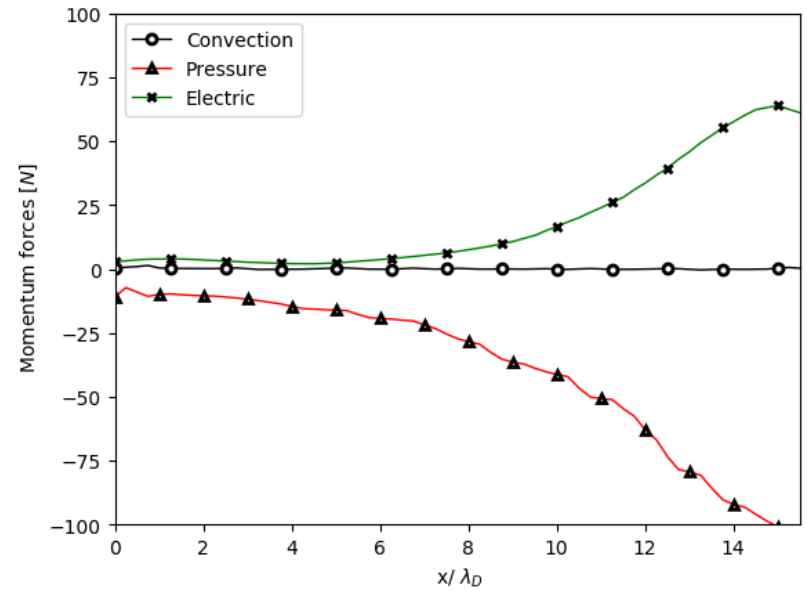

Figure 3 Balance between momentum equation forces inside the sheath at $t=0.3 \mu s$, AVIP-PIC simulation

the electrode supplies the electric circuit and creates a current. Away from the sheath the balance between these forces still play a role but the electric field is much reduced as the charge difference is smaller.

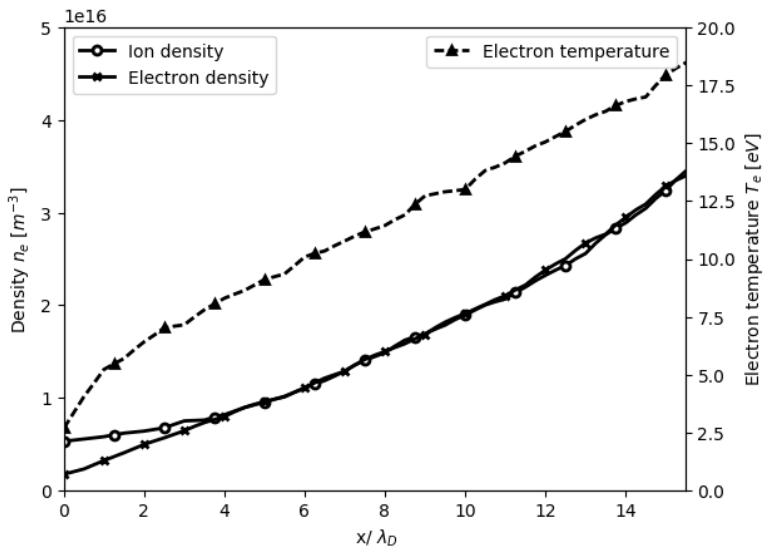

(a) Densities and electron temperature

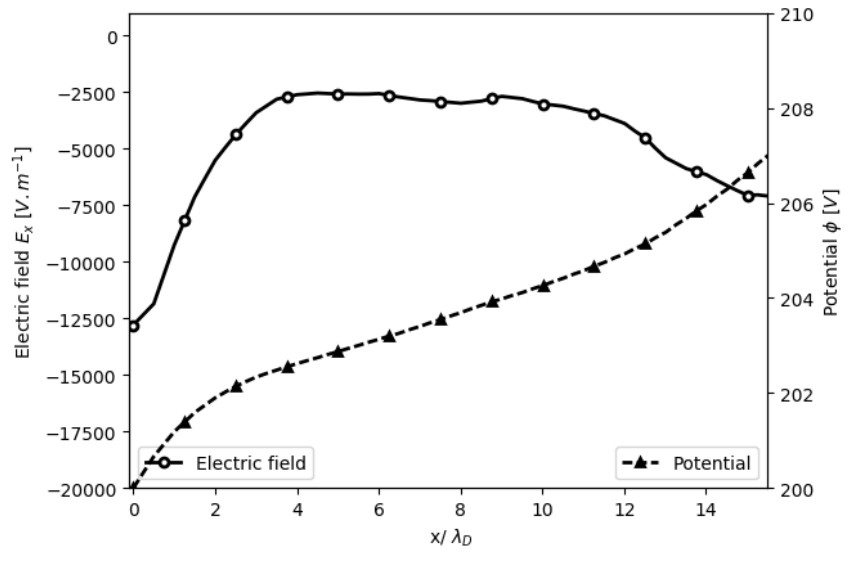

(b) Potential and electric field

Figure 4 Characteristics of the plasma inside the anode sheath at $t=0.3 \mu \mathrm{s}$, AVIP-PIC simulation

At equilibrium, Fig. 4a shows that the sheath thickness is strongly reduced down to 4 Debye lengths. In the present configuration, it leads to a sheath thickness of around $0.4 \mathrm{~mm}$. Note that he collisionless Child-Langmuir model (Eq. 35) based on fluid approximations, with an electron temperature of $T_{e}=10 \mathrm{eV}$ and a potential of $\phi_{0}=200 \mathrm{~V}$, predicts a sheath thickness of $s_{t h}=7.5 \lambda_{D}$.

The electron temperature decreases in the vicinity of the wall due to particles that leave the domain. In Fig. $4 \mathrm{~b}$, the maximum of electric field is located at the wall and is around $-12,500 \mathrm{~V} / \mathrm{m}$. The value is lower than in the transient state and no longer compensates for the loss of electron temperature at the wall. A potential drop of $3 \mathrm{eV}$ is also observed at the sheath edge.

In Fig. 5, the Bohm criterion (Eq. 34) is verified : the mean ion velocity is well equal to the ion sound speed at the beginning of the sheath and assures a stabilized sheath. At the boundary, the ion velocity is twice larger than the ion sound speed. As expected ions are supersonic and should be treated with caution in the fluid formalism.

\section{B. Wall boundary conditions for 10-moment fluid solvers}

Reproducing sheaths in fluid solvers is a known difficulty. The configuration previously studied with AVIP-PIC is now computed with AVIP-Fluid. A refined mesh with 100 cells per Debye length in the sheath area is used which 


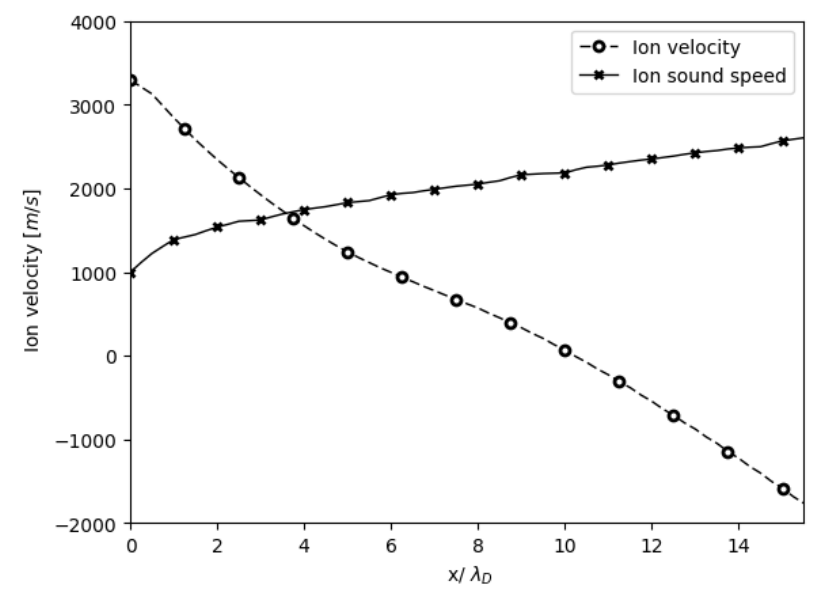

Figure 5 Bohm criterion : ion velocity compared to ion sound speed, AVIP-PIC simulation

corresponds to a cell size of approximately $1 \mu m$ near the wall. The HLLC Riemann solver is applied with a CFL number of 0.1. In these conditions, we suppose that numerical errors are negligible and that the results depend only on the boundary methodology and the physical model.

Two different types of boundary conditions are used and tested at the anode.

First, the vacuum condition is implemented as in the paper of Cagas [16]. Numerically, the Riemann solver at the boundary condition computes a self-consistent flux between two fluxes : a zero density and pressure flux corresponding to a vacuum on one side (called the "ghost cell"), and the flux predicted by the scheme on the other side. The solution at the boundary condition adjusts itself to compute the surface flux adapted to the predicted variables. To avoid numerical instabilities, the $p=0$ condition is not imposed in a hard way, but through a relaxation procedure :

$$
p_{\text {ghostcell }}=\frac{p_{\text {in }}}{100}
$$

where $p_{i n}$ is the predicted pressure at the boundary before the boundary condition procedure. The factor $\eta_{p}=1 / 100$ is arbitrary in the model and will be discussed later.

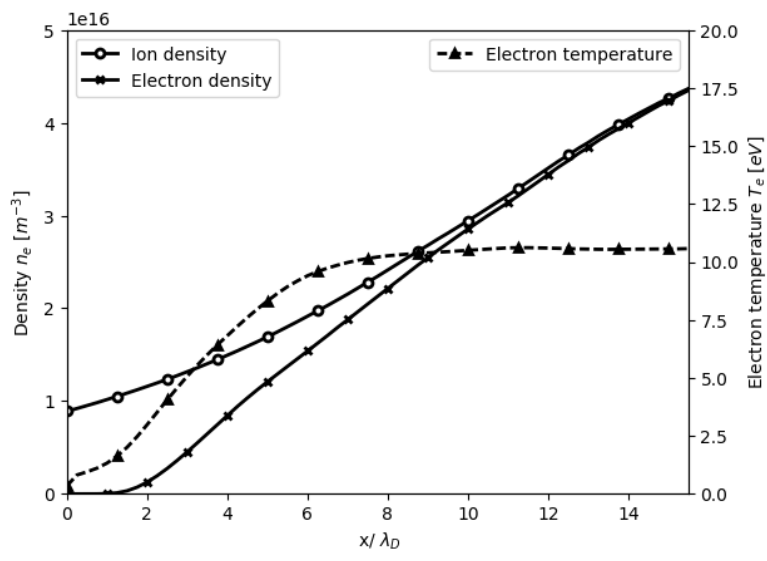

(a) Densities and electron temperature

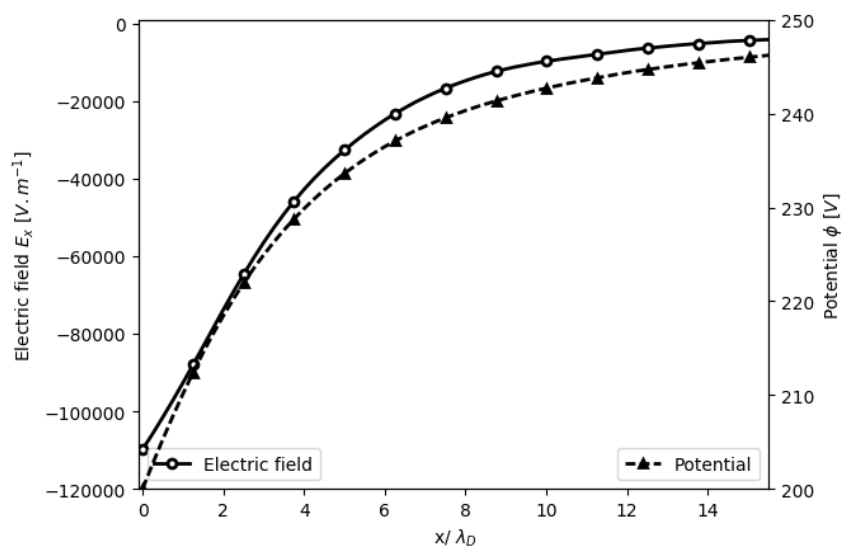

(b) Potential and electric field

Figure 6 Characteristics of the plasma inside the anode sheath at $t=0.3 \mu \mathrm{s}$, AVIP-Fluid simulation with vacuum condition

Figure 6 shows results with the vacuum condition at $t=0.3 \mu \mathrm{s}$. The transient phase is not presented here as it is similar to the PIC simulation. On Fig. 6b the potential drop is overestimated and reaches $40 \mathrm{~V}$ at the exit of the sheath. Consequently the electric field is higher inside the sheath and reaches $-110,000 \mathrm{~V} / \mathrm{m}$, which is 9 times the value of 
the PIC simulation. This huge electric field induces a larger sheath. On Fig. 6a, the steady sheath thickness is equal to around 10 Debye lengths, i.e., largely overestimated when compared to AVIP-PIC results. The sheath is also larger than predicted by the Child-Langmuir theory. In fact, the electron density induced by the vacuum condition is lower at the wall and leads to a difference of charge densities.

The electron temperature decreases inside the sheath and is however correct according to PIC results of Fig. 4 . A sensitivity study showed no impact of the relaxation ratio $\eta_{p}$ on these results.

The second boundary condition specifies the thermal flux at the boundary. The thermal velocity represents the mean thermal motion of particles $\alpha$ :

$$
v_{t h, \alpha}=\sqrt{\frac{2 k_{B} T_{\alpha}}{m_{\alpha}}}
$$

The thermal flux does not impact the mean particle velocity, i.e., the convective flux, but applies to the mass conservation [44]:

$$
F_{t h, \alpha}^{0}=n_{\alpha, i n} \frac{v_{t h, \alpha}}{2 \sqrt{\pi}}
$$

with $n_{\alpha, i n}$ the density predicted by the scheme inside the domain and $v_{t h, \alpha}$ the thermal velocity of the species $\alpha$. This flux represents the particles that leave the domain because of their velocity fluctuation and can be derived from the Maxwellian distribution function, see [43]. Considering that the mass flux leaving the domain is at the temperature computed inside the domain $T_{\alpha, \text { in }}$, the corresponding flux for the energy conservation reads :

$$
F_{t h, \alpha}^{2}=F_{t h, \alpha}^{0} \cdot \frac{k_{B} T_{\alpha, i n}}{m_{\alpha}}
$$

The components $F_{t h, \alpha}^{\gamma}$ are applied to the corresponding equation of $200-25$. Moreover the flux predicted by the scheme inside the domain is kept if the wall normal component of the velocity is positive. If the wall normal velocity component is negative, meaning an incoming flux, the flux is set to zero. This can be synthesized as :

$$
F_{b n d y, \alpha}^{\gamma} \cdot \vec{n}_{\perp}=\max \left(F_{i n, \alpha}^{\gamma} \cdot \vec{n}_{\perp}, 0\right)+F_{t h, \alpha}^{\gamma}
$$

where $\vec{n}_{\perp}$ is the boundary normal vector .

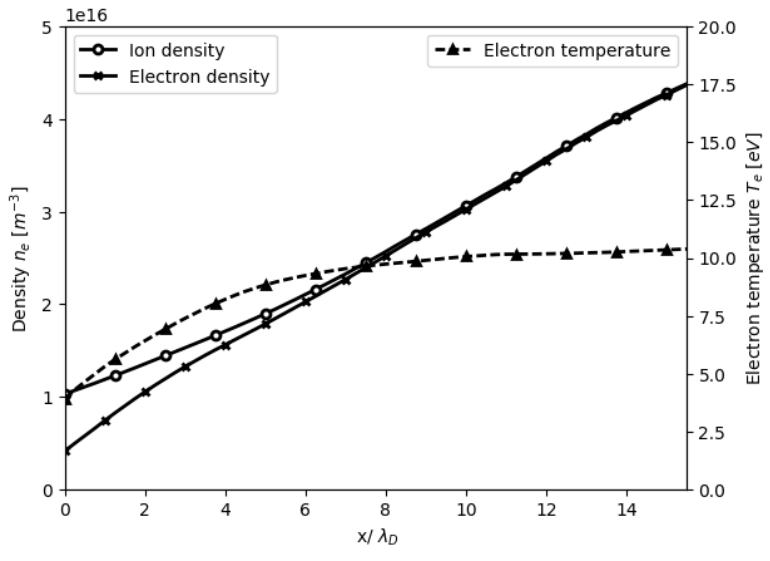

(a) Densities and electron temperature

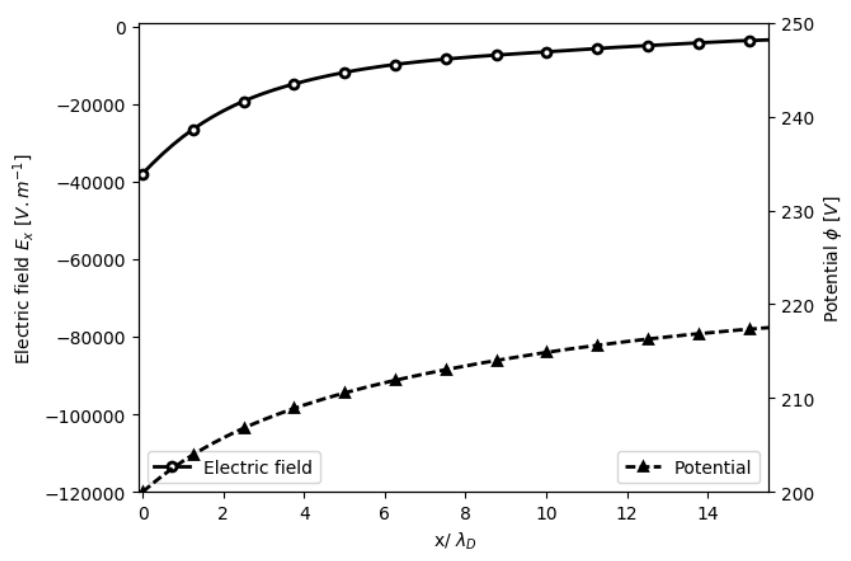

(b) Potential and electric field

Figure 7 Characteristics of the plasma inside the anode sheath at $t=0.3 \mu \mathrm{s}$, AVIP-Fluid simulation with thermal flux condition

Results obtained for the thermal flux condition at $t=0.3 \mu \mathrm{s}$, are shown in Fig. 77 The sheath thickness is around 6 Debye lengths which is in better agreement with AVIP-PIC results. The electron density at the wall boundary is much higher compared to the vacuum condition and corresponds to PIC results. This leads to a lower difference of 
charge density. Consequently, the potential drop is equal to $10 \mathrm{eV}$ and is slightly overestimated but closer to PIC results compared to the vacuum condition. Note that this drop has not yet reached a steady value as the transient regime is a bit longer for fluid simulations. Here, the electric field maximum is around $-38,000 \mathrm{~V} / \mathrm{m}$, much lower than for the vacuum condition. Finally the temperature drops in the sheath thickness and is in agreement with both previous simulations.

As explained in Section $\mathrm{A}$ the sheath reaches a steady state thanks to the balance between pressure gradient and Lorentz force. The analysis of these forces for the two different fluid boundary conditions is made at $t=0.3 \mu \mathrm{s}$ on Fig. 8

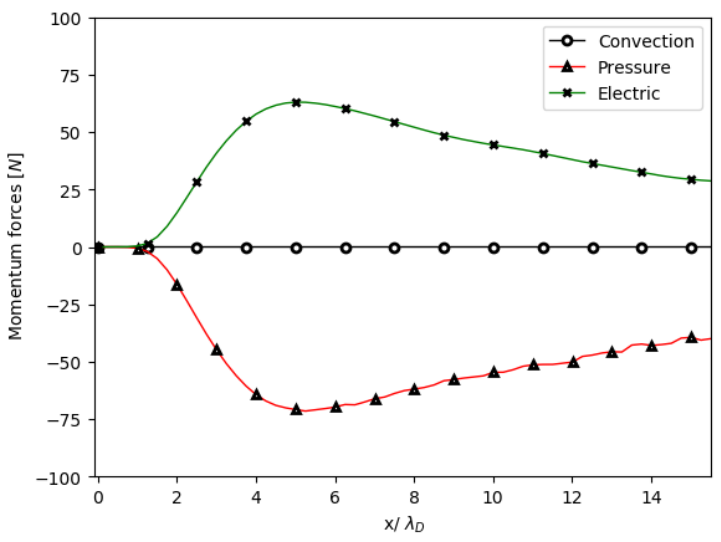

(a) Vacuum condition

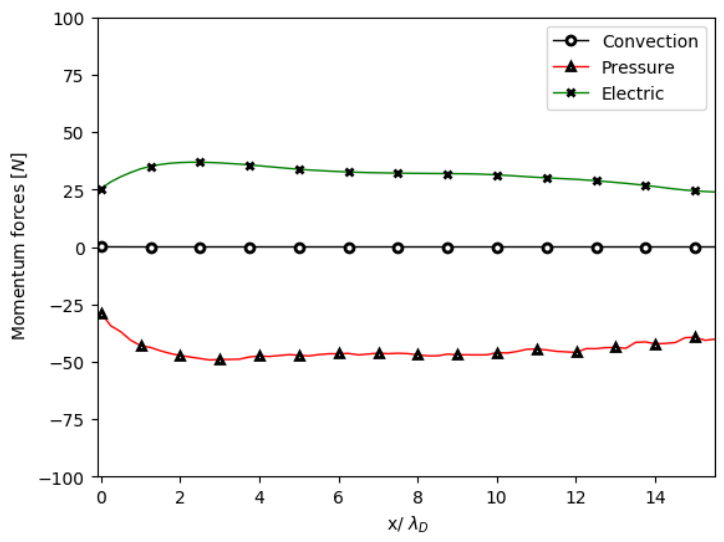

(b) Thermal flux condition

Figure 8 Balance between electron momentum equation forces inside the sheath at $t=0.3 \mu s$, AVIP-Fluid simulation

For the vacuum condition, Fig. 8a, we observe a zero value of all momentum forces at the wall. Imposing a zero pressure outside the domain leads to a zero pressure on the two first Debye lengths inside the domain by continuity. The pressure gradient is close to zero such as the electric contribution to respect the force balance inside the sheath. This also explains the low sensitivity of the results to the pressure ratio $\eta_{p}$, which only drives the convergence rate to the steady state but does not change the final pressure inside the domain. For the thermal flux condition, Fig. $8 \mathrm{~b}$, the sum of momentum forces at the wall is negative, around $2 \mathrm{~N}$. Electrons leave the domain as already shown in PIC results. The pressure gradient is not null but results from the predicted flux.

It is clear from these results that the vacuum condition properties are not conceptually similar to the PIC boundary conditions. The low electron density imposed at the boundary because of the vacuum pressure creates a larger sheath than the PIC simulation.The thermal flux condition, intuited from the microscopic particle behavior at the wall, is more suited to match PIC results.

In the present problem, the Debye length (Eq. 32) appears to be a key quantity which controls main sheath properties. On Fig. 9, the Debye length is plotted in the vicinity of the wall and compared to the AVIP-PIC results. The Debye length slightly increases inside the sheath because of a lower electron density. The thermal flux condition shows a very good evaluation of the Debye length in the sheath/presheath area, whereas the vacuum condition leads to large errors in the sheath region and close to the wall. This is to be related to the too low imposed electron pressure and consequently too low density at the wall.

Finally the Bohm criterion theory is evaluated in Fig. 10 Ion velocities for AVIP-Fluid with the two proposed boundary conditions, also applied for ions, are normalized by ion sound velocities and compared to the AVIP-PIC results. The ratio of ion velocity to ion sound speed of the AVIP-Fluid with the thermal flux condition is closer to the AVIP-PIC profile than the AVIP-Fluid with vacuum condition. Inside the presheath, the ion velocity is however slightly overestimated. The Bohm criterion is respected for the thermal flux condition : the sheath thickness (estimated at around 6 Debye lengths) corresponds to the value of ion velocity equality. However, the Bohm criterion is not valid for the AVIP-Fluid with the vacuum condition. The sheath thickness was estimated around 10 Debye lengths in this case and the ion velocity is not over the ion sound speed between 5 and 10 Debye lengths from the wall. The ion velocity in the sheath is largely overestimated due to the strong pressure condition at the boundary.

Table 1 summarizes the results of each simulation with characteristic properties of the sheaths. 


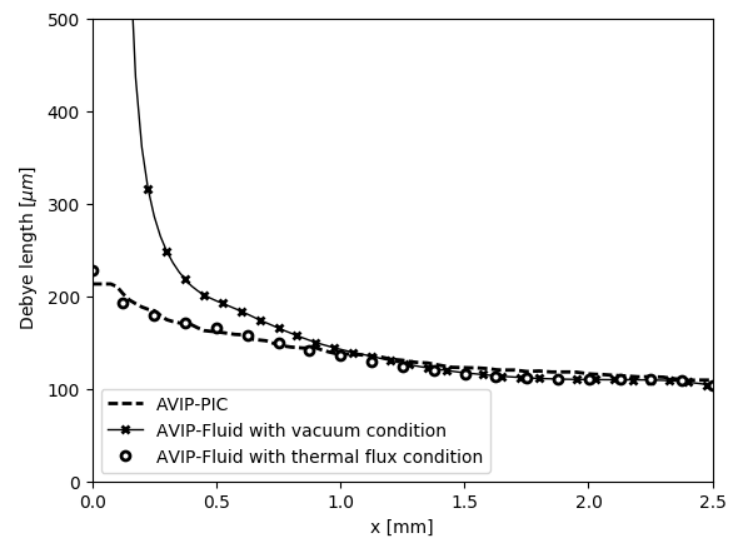

Figure 9 Debye length inside the domain for the various simulation cases

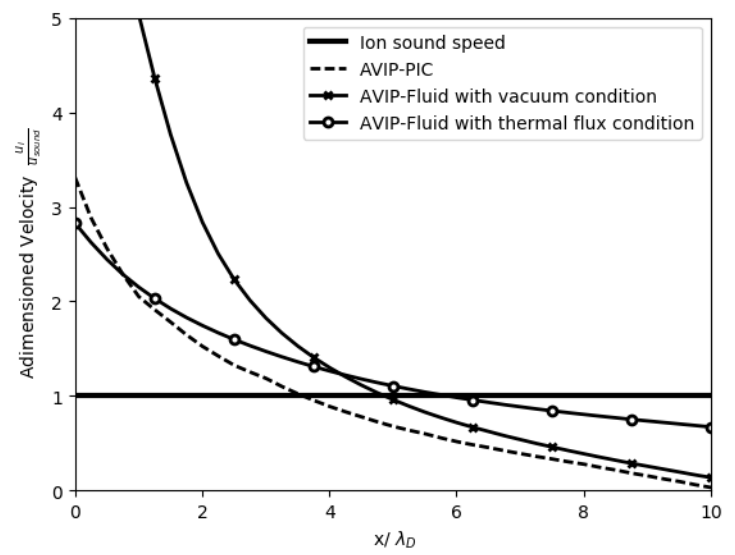

Figure 10 Bohm criterion : Ratio of the ion velocity to the ion sound speed for all simulation cases

TABLE 1 Characteristics of the sheath for all simulation cases at $t=0.3 \mu \mathrm{s}$

\begin{tabular}{|c|c|c|c|}
\hline Simulation case & sheath thickness $s$ & potential drop $\phi_{D}$ & Bohm criterion respected ? \\
\hline AVIP-PIC & $4 \lambda_{D}$ & $2 \mathrm{~V}$ & yes \\
\hline AVIP-Fluid with vacuum condition & $10 \lambda_{D}$ & $40 \mathrm{~V}$ & no \\
\hline AVIP-Fluid with thermal flux condition & $6 \lambda_{D}$ & $10 \mathrm{~V}$ & yes \\
\hline Child-Langmuir law & $7.5 \lambda_{D}$ & $/$ & $/$ \\
\hline
\end{tabular}

\section{Influence of mesh resolution}

Meshing near-wall regions with 100 cells per Debye length is not a viable solution for the simulation of complex $3 \mathrm{D}$ configurations since this would lead to too small computational time steps. The spatial resolution in the sheath is therefore a key factor and is studied in this subsection. Two meshes with respectively 2 cells per Debye length and 8 cells per Debye length inside the sheath are tested. Note that the characteristic Debye length chosen here is estimated away from the sheath, and equal to $105.2 \mu \mathrm{m}$, see Fig. 9 . The HLLC Riemann solver of order one is used with the thermal flux boundary condition. Results with these two meshes are plotted on Fig. 11.

A too low resolution of the near-wall region generates a peak of electron temperature. With two cells per Debye length, the electron temperature reaches $120 \mathrm{eV}$ at $t=0.3 \mu \mathrm{s}$ and continues to grow. The sheath is not stable and much larger (more than 25 Debye lengths). The reason is that the Bohm criterion stability is not fulfilled because the high peak of electron temperature induces a higher ion sound speed. With 8 cells per Debye length in the near-wall region, the sheath is created with a slight heating of about $8 \mathrm{eV}$ and the sheath thickness is overpredicted (around 11 Debye 


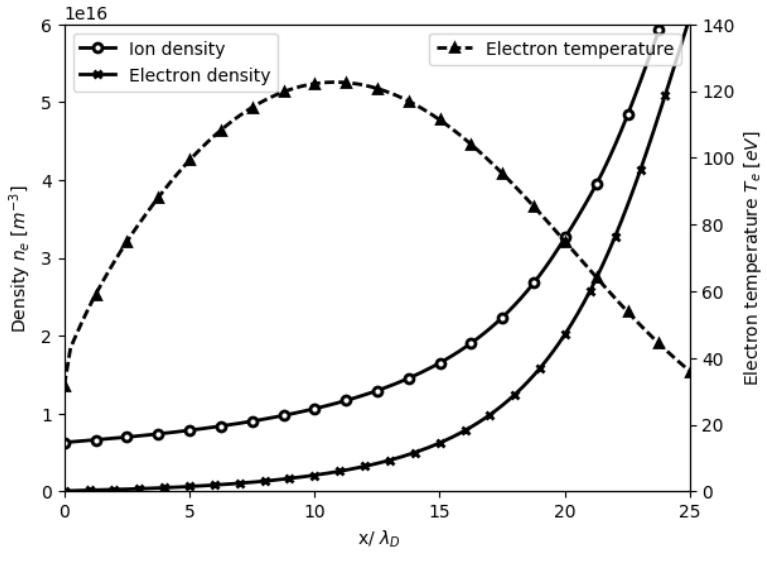

(a) 2 cells by Debye legnth

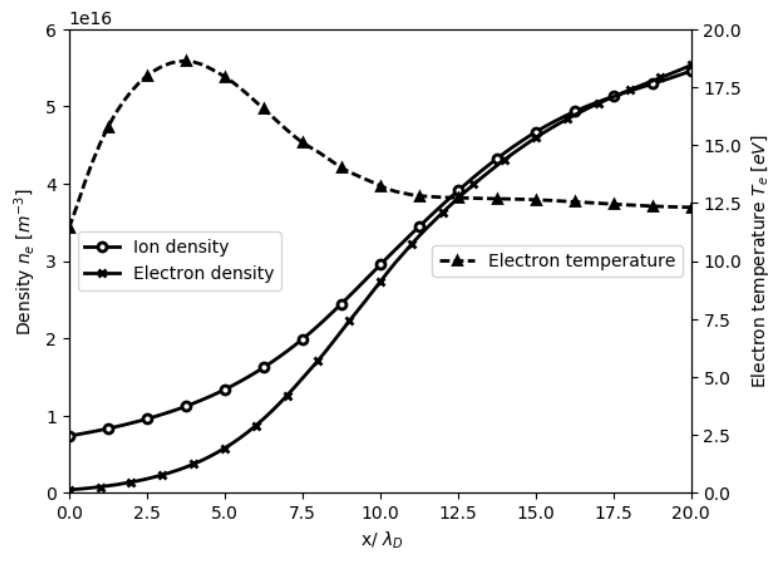

(b) 8 cells by Debye length

Figure 11 Densities and electron temperature of the plasma inside the anode sheath at $t=0.3 \mu \mathrm{s}$ for two different meshes, AVIP-Fluid simulations

lengths). From these results, the minimal resolution can be estimated at around 8 to 10 cells per Debye length. Note that this value is clearly dependent on the numerical scheme used (order 1 here). It means that an effort on the mesh resolution need to be done in order to correctly reproduce the sheath behavior near walls and electrodes.

\section{A 2D $r-z$ test case}

The physical model has been validated on simple test cases in order to assess of the plasma modeling, from standard plasma oscillation to detailed collision processes (not shown here for the sake of brevity). This final section presents the ability of the Fluid AVIP solver to simulate a Hall effect thruster configuration. For this purpose, the SPT 100 model which has been widely studied in the literature [45-47] is considered. A 2D r-z configuration of this thruster is chosen in order to get the global behavior of the plasma in the axial direction. Since the plume plays a role in the optimal operation of a Hall thruster, it is included in the computational domain. The main geometrical and operating parameters of the SPT 100 are referenced in the paper presented by Escobar [46] and are listed in Table 2.

TABLE 2 Main parameters of the SPT 100 for the $2 D$ r-z simulation

\begin{tabular}{|c|c|c|c|}
\hline$\dot{m}_{n}$ & $4.85 \mathrm{mg} / \mathrm{s}$ & $u_{0, n}$ & $300 \mathrm{~m} / \mathrm{s}$ \\
\hline$B_{\max }$ & $237 \mathrm{G}$ & $\phi_{a}$ & $300 \mathrm{~V}$ \\
\hline$L_{A C}$ & $33 \mathrm{~mm}$ & $L_{\text {channel }}$ & $25 \mathrm{~mm}$ \\
\hline$h_{\text {channel }}$ & $15 \mathrm{~mm}$ & $n_{0, e}$ & $10^{17} \mathrm{~m}^{-3}$ \\
\hline$T_{0, e}$ & $5 \mathrm{eV}$ & $T_{0, i}$ & $0.1 \mathrm{eV}$ \\
\hline
\end{tabular}

$\dot{m}_{n}$ is the Xenon mass flow rate at the anode and $u_{0, n}$ is the constant neutral velocity. The anode inside the Hall thruster has a fixed potential of $\phi_{a}=300 \mathrm{~V}$. The cathode, set to $\phi_{c}=0 \mathrm{~V}$ is positioned at a distance $L_{A C}$ at the right boundary of the domain in order to get discharge between two plates inside the chamber. A source term at $1 \mathrm{~mm}$ from the cathode injects electrons to compensate for the electronic current lost at the anode. The thermal flux boundary condition described in the previous section is used for ions and electrons. The axial magnetic field profile fits the SPT-100 magnetic configuration [46] and reaches a maximum $B_{\max }=237 \mathrm{G}$. Dimensions of the channel $L_{c h a n n e l}$ and $h_{\text {channel }}$ are described in Table 1 .

Figure 12 shows the axial profile of the initial neutral density corresponding to the background solution of the cited paper [46]. Ion and electron densities are initially constant and set to $n_{e, 0}=n_{i, 0}=10^{17} \mathrm{~m}^{-3}$. With such initial conditions, this leads to an initial Debye length of $\lambda_{D, 0}=52.6 \mu \mathrm{m}$.

The $2 \mathrm{D}$ mesh is made of 2, $2 M$ of triangles trying to respect the cell size recommendation made in Section $\mathrm{C}$ In the vicinity of walls, the cell size is fixed to $6 \mu \mathrm{m}$ in order to have around 9 cells per Debye length in the sheath. Inside the 


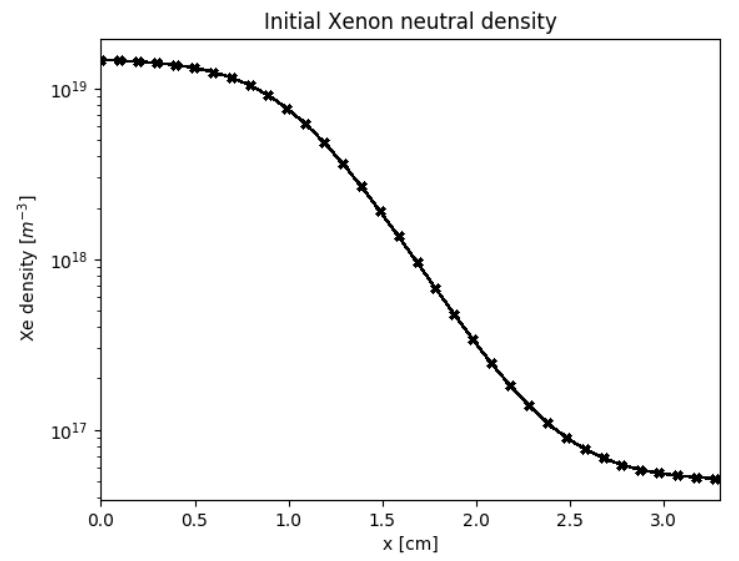

Figure 12 Initial Xenon neutral density corresponding to the background solution of Escobar paper [46]

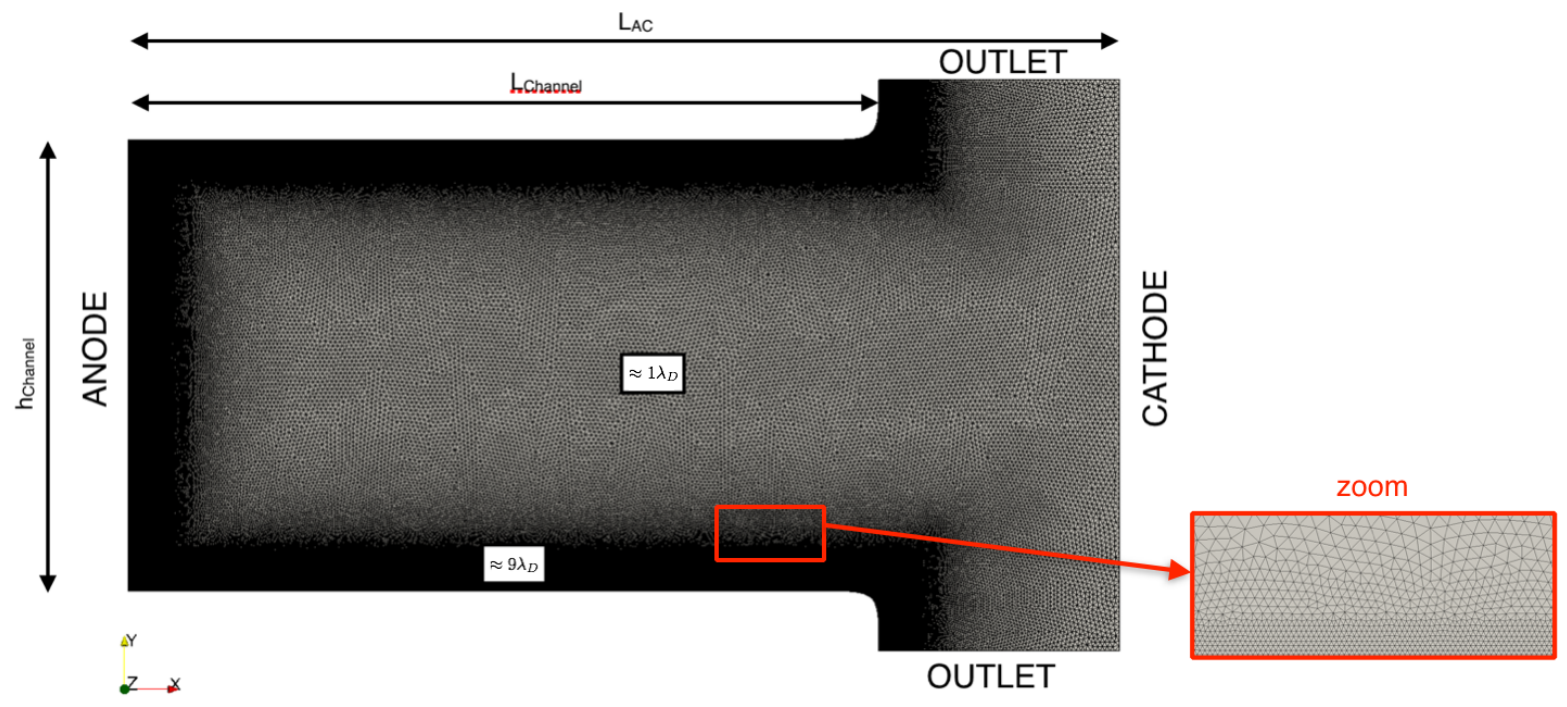

Figure 13 Mesh and geometry of the $r-z$ configuration

discharge chamber and away from the walls, cell sizes are around $50 \mu m$ to get 1 cell per Debye length.

Figure 14 shows preliminary results of the anode sheath for the r-z simulation after $0.1 \mu \mathrm{s}$. The electron density decreases near wall boundaries to form a sheath with a thickness around $500 \mu \mathrm{m}$ which corresponds to 10 Debye lengths. The solution is not yet stationary due to the low ion mobility. However it is possible to compute a theoretical thickness using the drift-diffusion law. For a potential of $300 \mathrm{~V}$ and an electron temperature of $5 \mathrm{eV}$, the theoretical thickness is around $s_{t h}=17 \lambda_{D}$. Here, the magnetic field and collision processes are not negligible inside the sheath and the Child-Langmuir law is not correct. Moreover, previous results in Section $\nabla$ have shown a slight overestimation of the analytical theory compared to PIC results. In Fig. 14 the electron temperature is overpredicted inside the sheath due to numerical thermal heating. Using a slightly more refined mesh at the wall should reduce this thermal heating at wall boundaries.

In Fig. 15. preliminary results of plasma properties inside the discharge chamber and the plume are shown at $t=0.1 \mu \mathrm{s}$. The formation of sheaths near chamber walls is visible on the electron density, see Fig. 15a. At the exit plane, in Fig. 15b the axial electric field is maximal leading to a strong acceleration of Xenon ions. This phenomena is standard in Hall effect thrusters and is responsible for the engine thrust. 


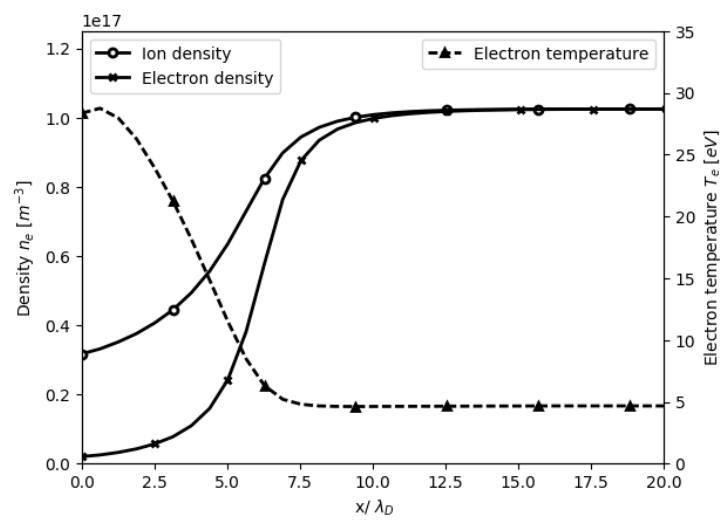

Figure 14 Densities and electron temperature inside the anode sheath at $t=0.1 \mu$ s for the r-z configuration

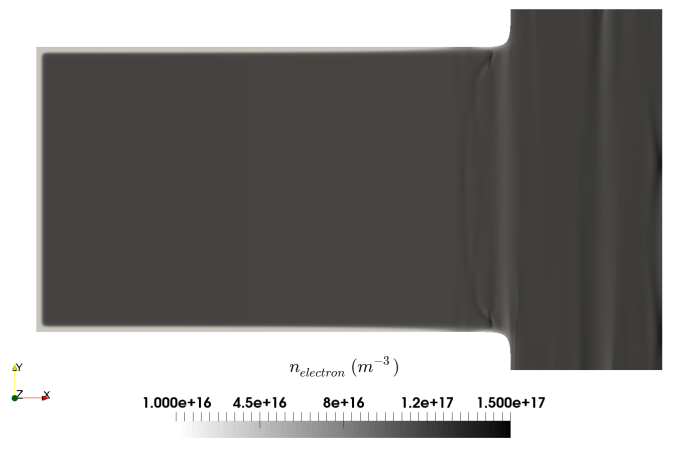

(a) Electron density

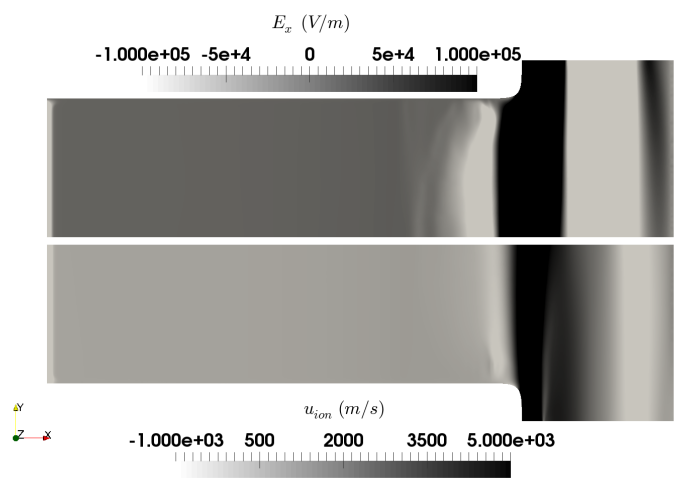

(b) Axial electric field and ion velocity

Figure 15 Plasma characteristics inside the Hall thruster chamber at $t=0.1 \mu \mathrm{s}$

\section{Conclusion}

AVIP is a 3D unstructured parallel-efficient solver developed to simulate bounded plasmas in Hall thruster configurations. The AVIP-Fluid model takes into account separated fluid entities for ions, electrons and neutrals with inertia and convection effects, elastic and inelastic collision processes. The equation system is closed using a Fourier law for the heat flux and coupled to the Poisson equation for the electric field resolution. The numerical resolution of these equations is done with a finite volume method coupled to a Riemann solver for convection terms adapted to unstructured meshes. Source term integration is done with an open source implicit solver. The resolution of the Poisson equation is adapted to highly parallel computation using the MAPHYS solver.

Inside Hall effect thrusters, the behavior of sheaths plays a crucial role in the thruster efficiency and lifetime. The study of sheaths raises the issue of explicit fluid boundary conditions. The vacuum condition presented in the paper of Cagas [16] fails to recover sheath properties compared to PIC results. The thermal flux condition is more in adequacy with PIC results, as it takes into account a truncated distribution function at the boundary condition. The mesh resolution is also a key point to obtain accurate sheath behavior without numerical thermal heating. The minimal mesh resolution for a numerical scheme of order one, in the vicinity of walls, is estimated to 8 cells per Debye length.

Finally the sheath theory is verified on a more realistic configuration of a Hall thruster. A 2D r-z simulation of a SPT 100 is performed on a mesh satisfying previous recommendations. Sheaths are formed near walls with a thickness around 10 Debye lengths. Preliminary results show the global behavior of the plasma characteristics at the exit plane, i.e., a strong electric field and a high ion velocity.

\section{Acknowledgments}

This work is financially supported by Safran Aircraft Engines (former SNECMA) as a part of a CIFRE convention and supervised by 
Stephan Zurbach. This work is also financially supported by the ANR chaire industrielle POSEIDON (ANR-16-CHIN-0003-01) (LPP/CERFACS/SAFRAN). It was performed using HPC resources from GENCI(Grant A0032B10157). The authors thank the fruitful collaboration with the Hiepacs team at INRIA Bordeaux for the Maphys solver. They also would like to acknowledge Pascal Chabert, Trevor Lafleur, Gerjan Hagelaar, Jean-Pierre Boeuf, Willca Villafana and all LPP Ph.D. students for useful discussions.

\section{Références}

[1] Hagelaar, G., Bareilles, J., Garrigues, L., and Boeuf, J., "Role of anomalous electron transport in a stationary plasma thruster simulation," Journal of Applied Physics, Vol. 93, No. 1, 2003, pp. 67-75.

[2] Bareilles, J., Hagelaar, G., Garrigues, L., Boniface, C., Boeuf, J., and Gascon, N., "Critical assessment of a two-dimensional hybrid Hall thruster model : Comparisons with experiments,” Physics of Plasmas, Vol. 11, No. 6, 2004, pp. 3035-3046.

[3] Lam, C., Fernandez, E., and Capelli, M., "A 2-D Hybrid Hall Thruster Simulation That Resolves the ExB Electron Drift Direction," IEEE Transactions on plasma science, Vol. 43, No. 1, 2015, pp. 86-94.

[4] Birdsall, C., "Particle-in-cell charged-particle simulations, plus Monte Carlo collisions with neutral atoms, PIC-MCC," IEEE Transactions on plasma science, Vol. 19, No. 2, 1991.

[5] Taccogna, F., Schneider, R., Longo, S., and Capitelli, M., "Kinetic simulations of a plasma thruster," Plasma Sources Science and Technology, Vol. 17, No. 2, 2008, p. 024003.

[6] Shumlak, U., and Loverich, J., "Approximate Riemann solver for the two-fluid plasma model," Journal of Computational Physics, Vol. 187, No. 2, 2003, pp. 620-638.

[7] Loverich, J., and Shumlak, U., "A discontinuous Galerkin method for the full two-fluid plasma model," Computer Physics Communications, Vol. 169, No. 1, 2005, pp. 251-255.

[8] Hakim, A., "Extended MHD Modelling with the Ten-Moment Equations," Journal Fusion Energy, Vol. 27 :36-43, 2008.

[9] Futtersack, R., "Modélisation fluide du transport magnétisé dans les plasmas froids,” Ph.D. thesis, Université de Toulouse, 2014.

[10] Alvarez Laguna, A., Ozak, N., Lani, A., Deconinck, H., and Poedts, S., "Fully-implicit finite volume method for the ideal two-fluid plasma model," Computer Physics Communications, 2018.

[11] Benilov, M. S., "Momentum and energy exchange between species of a multicomponent gas mixture due to inelastic and reactive collisions," Physics of Plasmas, Vol. 3, No. 7, 1996, pp. 2805-2812.

[12] Benilov, M. S., "A kinetic derivation of multifluid equations for multispecies nonequilibrium mixtures of reacting gases," Physics of Plasmas, Vol. 4, No. 3, 1997, pp. 521-527.

[13] Dujko, S., Markosyan, A., White, R., and Ebert, U., "High-order fluid model for streamer discharges : I. Derivation of model and transport data," Journal of Physics D : Applied Physics, Vol. 46, 2013, p. 475202.

[14] Markosyan, A., Dujko, S., and Ebert, U., "High-order fluid model for streamer discharges : II. Numerical solution and investigation of planar fronts," Journal of Physics D : Applied Physics, Vol. 46, 2013, p. 475203.

[15] Hagelaar, G., "Modeling methods for low-temperature plasmas," French accreditation to supervise research, Laboratoire Plasma et Conversion d'Energie LAPLACE, 2008.

[16] Cagas, P., Hakim, A., Juno, J., and Srinivasan, B., "Continuum Kinetic and multi-fluid simulations of classical sheaths," Physics of Plasmas, Vol. 24, No. 022118, 2017, pp. 1-11.

[17] Chen, G., and Raja, L. L., "Fluid modeling of electron heaheat in low-pressure, high frequency cacapacitive coupled plasma discharges," Journal of Applied Physics, Vol. 96, No. 11, 2004, pp. 6073-6081.

[18] Barral, S., "Numerical studies of Hall thrusters based on fluid equations for plasma," Ph.D. thesis, Polska Akademia Nauk, 2003.

[19] Escobar, E., Ahedo, E., and Parra, F., "On conditions at the sheath boundaries of a quasineutral code for Hall thrusters," Proc. 29th International Electric Propulsion Conference, Princeton, USA, IEPC-2005-041. Electric Rocket Propulsion Society, Fairview Park, $\mathrm{OH}$, Vol. 041, 2005, pp. 1-10.

[20] Gourdain, N., Gicquel, L., Montagnac, M., Vermorel, O., Gazaix, M., Staffelbach, G., Garcia, M., Boussuge, J.-F., and Poinsot, T., "High performance parallel computing of flows in complex geometries : I. Methods," Computational Science \& Discovery, Vol. 2, No. 015003, 2009, pp. 1-26. 
[21] Moureau, V., Domingo, P., and Vervisch, L., "Design of a massively parallel CFD code for complex geometries," Comptes Rendus Mecanique, Vol. 339, 2011, pp. 141-148.

[22] Karypis, G., and Kumar, V., "MeTis : Unstructured Graph Partitioning and Sparse Matrix Ordering System, Version 4.0," http://www.cs. umn. edu/ metis 2009. University of Minnesota, Minneapolis, MN.

[23] Abgrall, R., and Kumar, H., "Robust Finite Volume Schemes for Two-Fluid Plasma Equations," Journal of Scientific Computing, Vol. 60, No. 3, 2014, pp. 584-611.

[24] Toro, E., Spruce, M., and Speares, W., "Restoration of the contact surface in the HLL-Riemann solver," Shock Waves Springer-Verlag, Vol. 4, No. 25-34, 1994.

[25] Hindmarsh, A., "ODEPACK, A Systematized Collection of ODE Solvers," Scientific Computing, Vol. vol. 1 of IMACS Transactions on Scientific Computation, R. S. Stepleman et al, 1983, north-holland, amsterdam ed., pp. 55-64.

[26] Agullo, E., Giraud, L., and Poirel, L., "Robust coarse spaces for Abstract Schwarz preconditioners via generalized eigenproblems," Research Report RR-8978, INRIA Bordeaux, Nov 2016. URL https://hal.inria.fr/hal-01399203

[27] Agullo, E., Giraud, L., Houzeaux, G., Kuhn, M., Marait, G., and Poirel, L., "On the parallel design of coarse space corrections for sparse hybrid solvers," Research report, INRIA Bordeaux, 2018.

[28] Sydorenko, D., Smolyakov, A., Kaganovich, I., and Raitses, Y., "Plasma-sheath instability in Hall thrusters due to periodic modulation of the energy of secondary electrons in cyclotron motion," Physics of Plasmas, Vol. 15, No. 053506, 2008.

[29] Minelli, P., and Taccogna, F., "How to Build PIC-MCC Models for Hall Microthrusters," IEEE Transactions on plasma science, 2017, pp. 1-6.

[30] Anderson, N., and Walsh, G. R., "The formation of a plasma sheath," International Journal of Electronics, Vol. 31, No. 3, 1971, pp. 217-222.

[31] Takamura, S., "Sheath formation in a plasma with energetic electrons," Physics Letters A, Vol. 133, No. 6, 1988.

[32] Scheiner, B., Baalrud, S., Yee, B. T., Hopkins, M. M., and V., B. E., “Theory of the Electron Sheath and Presheath,” Physics of Plasmas, Vol. 22, No. 123520, 2015, pp. 1-12.

[33] Langmuir, I., "The effect of space charge and initial velocities on the potential distribution and thermionic current between plane electrodes," Physical Review, Vol. 21, No. 954, 1923.

[34] Riemann, K., "The Bohm criterion and sheath formation," Journal of Physics D : Applied Physics, Vol. 24, 1991, pp. $493-518$.

[35] Lieberman, M. A., and Lichtenberg, A., Principles of plasmas Discharges and materials processing, John Wiley \& Sons, 1994.

[36] Chabert, P., and Braithwaite, N., Physics of Radio-Frequency plasmas, Cambridge Univ Press, 2011.

[37] Ahedo, E., "Presheath/sheath model with secondary electron emission from two parallel walls," Physics of Plasmas, Vol. 9, No. 10, 2002, pp. 4340-4347.

[38] Hofer, R., Mikellides, I., Katz, I., and Goebel, D., "Wall sheath and electron mobility modeling in hybrid-PIC Hall thruster simulations," AIAA Paper, Vol. 5267, 2007, p. 2007.

[39] Hobbs, G., and Wesson, J. A., "Heat flow through a Langmuir sheath in the presence of electron emission," Plasma Physics, Vol. 9, 1967, pp. 85-87.

[40] Stephens, K. F., and Ordonez, C. A., "Sheath and presheath potentials for anode, cathode and floating plasma-facing surfaces," Journal of Applied Physics, Vol. 85, No. 5, 1999, pp. 2522-2527.

[41] Robertson, S., "Sheaths in laboratory and space plasmas," Plasma Phys. Control. Fusion, Vol. 55, No. $093001,2013$.

[42] Hagelaar, G., De Hoog, F., and Kroesen, G., "Boundary conditions in fluid models of gas discharges," Physical Review E, Vol. 62, No. 1, 2000, pp. 1452-1454.

[43] Hagelaar, G., Fubiani, G., and Boeuf, J., "Model of an inductively coupled negative ion source : I. General model description," Plasma Sources Sci. Technol., Vol. 20, No. 015001, 2011.

[44] Becker, M., Kahlert, H., Sun, A., Bonitz, M., and Loffhagen, D., "Advanced fluid modeling and PIC/MCC simulations of low-pressure CCRF discharges,” Plasma Sources Sci. Technol., Vol. 26, 2017, p. 044001. 
[45] Sullivan, K., Fox, J., Martinez-Sanchez, M., and Batischev, O., "Kinetic Study of Wall Effects in SPT Hall Thrusters," 40 th AIAA/ASME/SAE/ASEE Joint Propulsion Conference and Exhibit, 2004, pp. 1-10.

[46] Escobar, D., and Ahedo, E., "Global stability analysis of azimuthal oscillations in Hall Thrusters," 33rd International Electric Propulsion Conference, Vol. 304, 2013, pp. 1-14.

[47] Boeuf, J. P., “Tutorial : Physics and modeling of Hall thrusters,” Journal of Applied Physics, Vol. 121, No. 011101, 2017, pp. $1-24$. 